\title{
MicroRNA-409-3p Functions as a Tumor Suppressor in Human Lung Adenocarcinoma by Targeting c-Met
}

\author{
Li Wan $^{\mathrm{a}}$ Lingjun Zhu ${ }^{\mathrm{b}}$ Jianghao Xua, Binbin Lu ${ }^{\mathrm{a}} \quad$ Yucai Yang ${ }^{\mathrm{a}}$ Fengzhen Liu ${ }^{\mathrm{a}}$ \\ Zhaoxia Wang ${ }^{a}$
}

aDepartment of Oncology, The Second Affiliated Hospital of Nanjing Medical University, Nanjing, bDepartment of Oncology, The First Affiliated Hospital of Nanjing Medical University, Nanjing, 'Department of Clinical Medicine, The First Medical college of Nanjing Medical University, Nanjing, P.R. China

\section{Key Words}

miR-409-3p • c-Met $\bullet$ prognosis $・$ Lung adenocarcinoma $・$ Tumor suppressor

\begin{abstract}
Background/Aims: Dysregulation of microRNAs is correlated with tumor development. The aim of this study is to investigate the clinicopathologic and prognostic significance of microRNA (miR)-409-3p and its tumor suppressor roles in lung adenocarcinoma (LAD). Methods: Quantitative real-time PCR ( $q R T-P C R$ ) was performed to detect miR-409-3p expression in LAD tissues and corresponding noncancerous tissues. Additionally, the correlations of miR409-3p expression with clinicopathologic factors and prognosis of patients were statistically analyzed. Next, we investigated whether miR-409-3p could function as a tumor suppressor in LAD cells via regulation of Akt signaling by targeting receptor tyrosine kinase (c-Met). Results: MiR-409-3p was significantly downregulated in LAD tissues compared with corresponding noncancerous tissues. Low miR-409-3p expression was observed to be significantly correlated with poorer tumor differentiation, advanced PTNM stage and higher incidence of lymph node metastasis. Multivariate Cox regression analyses showed that miR-409-3p expression was an independent prognostic factor for LAD patients. Functional analyses indicated that miR-409-3p could inhibit growth, induce apoptosis, reduce migration and invasion in LAD cells via inactivation of Akt signaling by targeting c-Met. Conclusions: MiR-409-3p was an independent prognostic factor and functioned as a tumor suppressor in LAD via regulation of Akt signaling by targeting c-Met.
\end{abstract}

Copyright @ 2014 S. Karger AG, Basel

L. Wan, L. Zhu and J Xu contributed equally to this work and should be regarded as joint first authors. 


\section{Introduction}

Lung cancer, one of the most common malignancies around the world, is the leading cause of cancer death worldwide [1]. In China, about 300,000 new lung cancer patients and more than 250,000 deaths from the disease are predicted each year. Adenocarcinoma of the lung is the most common type of lung cancer and accounts for 30 to 35 percent of primary lung tumors. Recent advances in the multidisciplinary management of the disease, including surgery, chemoradiotherapy and molecular-targeted therapy, but a substantial proportion of LAD patients with localized or locally advanced disease will eventually die [2]. Lung carcinogenesis is a complex multistep process involving genetic dysregulation of protooncogenes and tumor suppressor genes [3]. Therefore, a better understanding of molecular mechanisms underlying LAD development will contribute to identifying novel prognostic markers and molecular therapeutic targets for human LAD.

MicroRNAs (miRNAs), a class of small non-coding RNAs, can function posttranscriptionally through imperfect base pairing with specific sequences in the 3' untranslated regions (UTRs) of target mRNAs leading to transcript degradation or translational inhibition [4, 5]. Emerging evidence indicates that dysregulation of miRNAs is involved in many human biological and pathological processes, such as cell proliferation, differentiation, development, apoptosis, and tumorigenesis [6-8]. It has been revealed that miRNAs function in various stages of cancer development, showing that abnormal miRNA expressions play critical roles in modulating expression of known oncogenes or tumor suppressor genes during cancer progression [9]. Recently, the correlation of dysregulation of miRNAs with LAD is increasingly reported. By high-throughput sequencing combined with differential expression analysis, Xie' et al identified that 7 microRNAs were downregulated and 21 microRNAs were up-regulated in LAD with bone metastasis [10]. By performing microRNA expression profiles of whole blood in lung adenocarcinoma, Patnaik and his colleagues showed that four microRNAs (miR-190b, miR-630, miR-942, and miR1284) were the most frequent constituents of the classifiers generated during the analyses, suggesting that whole blood microRNA expression profiles can be used to distinguish lung cancer cases from clinically relevant controls [11]. Additionally, Zhang' et al reported that circulating miR-195 and miR-122 may have prognostic values in predicting the overall survival as well as predicting EGFR mutation status in non-smoking female patients with lung adenocarcinoma, suggesting that measuring plasma levels of miR-195 and miR-122 may especially be useful in EGFR mutant patients with lung adenocarcinoma [12]. These data suggest that dysregulation of miRNAs may play important roles in LAD progression and development. Previously, miR-409-3p has been reported to inhibit growth, invasion and metastasis of tumor cells, including bladder cancer, gastric cancer and fibrosarcoma [13-15]. However, expression of miR-409-3p and its clinicopathologic or prognostic significance in LAD are not fully understood. In addition, the roles of miR-409-3p in LAD development and its possible molecular mechanisms remain to be further elucidated.

In the present study, we show that downregulation of miR-409-3p in LAD is correlated with an aggressive phenotype and poor prognosis of patients. Further investigations indicated that miR-409-3p directly targeted the 3'-UTRs of receptor tyrosine kinase c-Met, which functions as an oncogene in LAD, to suppress its expression, which in turn led to the inhibition of growth or invasion in LAD cells. Thus, miR-409-3p is a good prognostic factor for LAD patients and functions as a tumor suppressor in human LADs.

\section{Marterials and Methods}

\section{Ethics statement}

Human LAD and matched non-cancerous tissues were collected after obtaining written informed consent from all patients. The study was approved by the Institutional Review Board of Nanjing Medical University. 
Cell lines and culture

A normal human bronchial epithelial cell line (HBE) and three LAD cell lines (A549, SPC-A1 and PC9) were purchased from Shanghai Institute Chinese academy of science and cultured in RPMI 1640 media (Invitrogen, CA, USA) supplemented with $10 \%$ fetal bovine serum (FBS) and $100 \mu \mathrm{M}$ each of penicillin and streptomycin in a humidified atmosphere of $5 \% \mathrm{CO}_{2}$ at $37^{\circ} \mathrm{C}$.

Patients and tissue samples

Fresh LAD and matched adjacent normal tissue specimens were collected from 128 patients who underwent surgery after removal of the necessary amount of tissue for routine pathology examination at the Department of Chest Surgery of the First or Second Affiliated Hospital of Nanjing Medical University. Samples were snap-frozen in liquid nitrogen and stored at $-80^{\circ} \mathrm{C}$ until RNA extraction. Diagnosis of LAD was determined according to the latest World Health Organization (WHO) criteria and TNM stage classification (UICC 2002). Related clinical data were collected, including gender, age, smoking history, tumor differentiation, pTNM stage, lymph node metastasis, and 5-year follow-up survival. All patients did not receive chemotherapy or radiotherapy prior to surgery. Patient characteristics are shown in Table 1. Pathologic staging was performed in accordance to the current International Union Against Cancer tumorlymph node-metastasis classification. Written informed consent was obtained from all patients. The ethics committee of Jiangsu Province Medical Association approved the study protocol.

\section{RNA isolation and qRT-PCR assay}

Total RNA was isolated from cells using Trizol (Invitrogen), and $2 \mu \mathrm{l} \mathrm{RNA}(1 \mu \mathrm{g} / \mu \mathrm{l})$ was used to synthesize cDNA with Super-Script II First-Strand Synthesis System (Invitrogen) or TaqMan ${ }^{\circledR}$ MicroRNA Reverse Transcription Kit (Applied Biosystems). Aliquots of the reaction mixture were used for real-time PCR with Power SYBR Green PCR Master Mix or with the TaqMan ${ }^{\circ} 2 \times$ Universal PCR Master Mix. The reaction conditions: $50^{\circ} \mathrm{C}$ for $20 \mathrm{~s}, 95^{\circ} \mathrm{C}$ for $10 \mathrm{~min}$ followed by 40 cycles of $95^{\circ} \mathrm{C}$ for $15 \mathrm{~s}, 60^{\circ} \mathrm{C}$ for $1 \mathrm{~min}$. We calculated a $\Delta \mathrm{Ct}$ (target-reference), which is equal to the difference between threshold cycles for miR409-3p (ie, target) and the threshold cycle for U6 RNA (ie, reference) (ie, $\Delta \mathrm{Ct}$ (target-reference) $=\mathrm{Ct}$ target-Ct reference). The fold-change between patient or cell sample and a normal control for miR-409-3p or c-Met was calculated with the $2^{-\Delta \Delta C t}$ method. All real-time PCR experiments were performed in triplicate.

Transfection of oligonucleotides and plasmid vectors

The pGCMV/EGFP/miRNA vectors with and without human miR-409-3p expression (pGCMV/ miR-409-3p and pGCMV/miR-NC), miR-409-3p mimics or inhibitor (anti-miR-409-3p) and their control oligonucleotides (anti-miR-NC) were obtained from Genepharma (Shanghai, China). For c-Met interfering, DNA template oligonucleotides corresponding to c-Met (accession number NM_000245) were synthesized as follows: c-Met-shRNA1 (sense, 5'-GATCCGCCCAAC-TACAGAAATGGTTTCAAGAGAACCATTTCTGTAGTTGGGCA GA-3'), c-Met-shRNA2 (sense, 5'-GATCCAAGTGCAGTATCCTCTGACTTCAAGAGAGTCAGAGGATACTGCACTTAGA-3') and a non-specific shRNA, control-shRNA (sense, 5'-GATCTAAGCATTAGGTACAGCATTTCAAGAGAATGCTGTACCTAATGCTTAGA-3'). All of the above sequences were inserted into the BglII and HindIII enzyme sites of pSUPER.retro vector, respectively. Those recombinant plasmids were named pSUPERshRNA/control (shRNA/control), pSUPER-shRNA/c-Met1(shRNA/c-Met1), and pSUPER-shRNA/c-Met2 (shRNA/c-Met2) vector, respectively. For ectopic expression of c-Met, the open reading frame of c-Met that was generated by PCR using the following primers: sense 5'-CGCTCGAGAGTGGGCAGAGGAATGCCTG-3'; reverse 5'-GCGGATCCGAAGAGGTCTGG-GGGACTGC-3', and then inserted into the pcDNA 3.1(+) expression vector which was named pcDNA/c-Met. Those recombinant vectors were confirmed by the digestion analysis of restriction endonuclease and all inserted sequences were verified by DNA sequencing. The transfection were performed using Lipofectamin $\mathrm{e}^{\mathrm{TM}} 2000$ (Invitrogen, USA) according to the instructions provided by the manufacturer. Stable cell lines expressing pri-miR-409-3p were selected with G418 (400 mg/mL) and colonies stably expressing shRNAs were selected with $8.0 \mu \mathrm{g} / \mathrm{ml}$ puromycin (Sigma, USA).

\section{Western blotting assay}

Cells or tissues were washed with cold phosphate-buffered saline solution, and total proteins were extracted in the extraction buffer (150mM sodium chloride; 50mMTris hydrochloride, pH7.5;1\%glycerol;

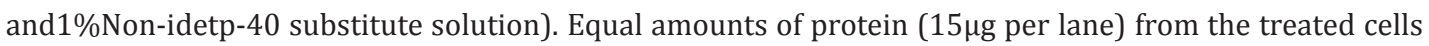


were loaded and electrophoresed on an 8\% sodium dodecyl sulfate (SDS) polyacrylamide gel and then electroblotted onto nitrocellulose membrane, blocked by 5\% skim milk, and probed with the antibodies to c-Met, phosphorylated c-Met (p-c-Met), cleaved caspase-3, total caspase-3, cleaved PARP, total PARP, phosphorylated AKT (Ser473), total Akt, Bcl-2, Bax, MMP-2, MMP-9 and GAPDH (Santa Cruz Biotechnology, Santa. Cruz, California, USA), followed by treatment with secondary antibody conjugated to horseradish peroxidase. The proteins were detected by the enhanced chemiluminescence system and exposed to x-ray film. Band density was measured by photoimage analysis using the Quantity one software (Bio-Rad, Hercules, CA, USA), and expressed as the percentage of density of the respective housekeeping GAPDH.

\section{3-(4,5-dimethylthazol-2-yl)-2,5-diphenyltetrazolium bromide (MTT) assay}

The cell viability was measured by MTT assay (Sigma, USA). In brief, the cells were seeded into five 96well culture plates with each plate having all four kinds of A549 cells transiently transfected with miR-409$3 p$ mimics (miR-NC mimics) or anti-miR-409-3p (anti-miR-NC) (6-parallel wells/group). On each day, 200 $\mu \mathrm{L}$ MTT $(5 \mathrm{mg} / \mathrm{mL})$ was added to each well, and the cells were incubated for at $37^{\circ} \mathrm{C}$ for additional $4 \mathrm{~h}$. Then the reaction was stopped by lysing the cells with $150 \mu \mathrm{L}$ DMSO for $5 \mathrm{~min}$. Optical densities were determined on a Versamax microplate reader (Molecular Devices, Sunnyvale, CA, USA) at $490 \mathrm{~nm}$.

\section{Colony formation assay}

The A549 cells stably transfected with pGCMV/miR-409-3p or pGCMV/miR-NC were trypsinized to single cell suspensions and were seeded 6-well plates at 500/well. After 14 days culture RPMI 1640 medium, the colonies were stained with Giemsa solution and the number of colonies was counted. Each experiment was performed in triplicate.

\section{Flow cytometric analysis of apoptosis}

The cells were harvested, washed twice with cold PBS, fixed in ice-cold $70 \%$ ethanol, and incubated overnight at $-20^{\circ} \mathrm{C}$. Then, cells were stained with $40 \mu \mathrm{g} / \mathrm{mL}$ of propidium iodide (PI) for $30 \mathrm{~min}$. The minimum of $1.0 \times 10^{6}$ cells were collected and analyzed by software Cell Quest (Becton Dickinson Co., NJ, USA). The percentage of cells with apoptotic nuclei (\% apoptosis) was calculated.

\section{Wound healing assay}

The cells were seeded into 24 -well tissue culture plates. $48 \mathrm{~h}$ later, an artificial homogenous wound was created onto the monolayer with a sterile plastic $100 \mu \mathrm{L}$ micropipette tip. After wounding, the debris was removed by washing the cells with serum-free medium. Migration of cells into the wound was observed at different time points. Cells that migrated into the wounded area or cells with extended protrusion from the border of the wound were visualized and photographed under an inverted microscope. A minimum of five randomly chosen areas were measured and the distance of cell migration to the wound area was determined.

\section{Transwell invasion assay}

Transwell invasion assays were performed with 6-well matrigel-coated chambers from BD Biosciences (Bedford, MA, USA). In brief, the cells were seeded into inserts at $05.0 \times 10^{3} /$ insert in serum-free medium and then transferred to wells filled with the culture medium containing $10 \% \mathrm{FBS}$ as a chemoattractant. After $24 \mathrm{~h}$ of incubation, non-invading cells on the top of the membrane were removed by scraping. Invaded cells on the bottom of the membrane were fixed, followed by staining with $0.05 \%$ crystal violet. The number of invaded cells on the membrane was then counted under a microscope.

\section{Luciferase activity assay}

To testify the validation of c-Met as a direct target of miR-409-3p, we performed miRNA target luciferase reporter assay using a pEZX-MT01 target reporter plasmid containing c-Met/3'-UTR (Genecoepia, Rockville, MD). Additionally, we generated mutant c-Met/3'-UTR reporter construct (pEZX-Luc-c-Met/3'-UTR-mut) by site-directed mutagenesis in the putative target site of miR-409-3p in the wild-type c-Met/3'-UTR (pEZX-Luc-c-Met/3'-UTR-wt) using Stratagene QuikChange ${ }^{\circledR}$ Site-Directed Mutagenesis Kit (Stratagene, Heidelberg, Germany). The cells were transiently cotransfected for $24 \mathrm{~h}$ with reporter plasmids (200 ng) and $100 \mathrm{nM}$ of miR-409-3p mimics or inhibitor and harvested in reporter lysis buffer. Both firefly luciferase 
Table 1. Correlation of miR-409-3p expression with clinicopathologic factors of LAD patients. ${ }^{*} P<0.05$ was considered statistically significant

\begin{tabular}{|c|c|c|c|}
\hline $\begin{array}{l}\text { Clinicopathologic } \\
\text { factors }\end{array}$ & $\begin{array}{l}\text { Low miR-409-3p } \\
\text { expression }(n=38) \\
\text { Number }(\%)\end{array}$ & $\begin{array}{l}\text { High miR-409-3p } \\
\text { expression }(n=46) \\
\text { Number }(\%)\end{array}$ & $P$-value \\
\hline Age (years) & & & 0.744 \\
\hline$<60$ & $16(42.1)$ & $21(45.7)$ & \\
\hline$\geq 60$ & $22(57.9)$ & $25(54.3)$ & \\
\hline Gender & & & 0.433 \\
\hline Male & $26(68.4)$ & $35(76.1)$ & \\
\hline Female & $12(31.6)$ & $11(23.9)$ & \\
\hline Smoking condition & & & 0.071 \\
\hline Nonsmokers & $20(52.6)$ & $33(71.7)$ & \\
\hline Smokers & $18(47.4)$ & $13(28.3)$ & \\
\hline Tumor differentiation & & & $0.032 *$ \\
\hline Well & $8(21.1)$ & $22(47.8)$ & \\
\hline Moderate & $15(39.5)$ & $14(30.4)$ & \\
\hline Poor & $15(39.4)$ & $10(21.8)$ & \\
\hline T-primary tumor & & & 0.112 \\
\hline $\mathrm{T} 1+\mathrm{T} 2$ & $20(52.6)$ & $32(69.6)$ & \\
\hline $\mathrm{T} 3+\mathrm{T} 4$ & $18(47.4)$ & $14(30.4)$ & \\
\hline Lymph node metastasis & & & $0.020^{*}$ \\
\hline Negative & $15(39.5)$ & $31(67.4)$ & \\
\hline Positive & $23(60.5)$ & $15(32.6)$ & \\
\hline pTNM stage & & & $0.028 *$ \\
\hline Stage I/II & $14(36.8)$ & $28(60.9)$ & \\
\hline Stage III/IV & $24(63.2)$ & $18(39.1)$ & \\
\hline Cancer-related death & & & 0.569 \\
\hline Alive & $11(28.9)$ & $16(34.8)$ & \\
\hline Death & $27(71.1)$ & $30(65.2)$ & \\
\hline
\end{tabular}

and Renilla luciferase activities were measured using the Dual-Luciferase assay kit (Promega, Madison, WI) according to manufacturer's instructions. The luciferase activity normalized against protein concentration was expressed as a ratio of firefly luciferase to Renilla luciferase unit.

\section{Statistical analysis}

Statistical analysis was performed with SPSS version 13.0 (SPSS Inc., Chicago, IL). Values are expressed as the mean \pm SD. The relationship between miR-409-3p expression and clinicopathologic factors was analyzed by Pearson's chi-squared test. Survival curves were plotted by the Kaplan-Meier method and compared using the log-rank test. Survival data were evaluated using univariate and multivariate Cox regression analyses. The correlation between the miR-409-3p expression level and the protein level of c-Met was evaluated by Spearman's rank correlation coefficients. $P<0.05$ was considered statistically significant.

\section{Results}

MiR-409-3p is downregulated in primary LAD tissues

First, qRT-PCR assay was performed to detect the expression levels of miR-409-3p in 34 pairs of human LAD tissues and their matched adjacent non-cancerous tissues, and the results indicated that miR-409-3p was significantly downregulated in $79.4 \%(27 / 34)$ of the LAD tissues examined in comparison with the matched adjacent non-cancerous tissues from the same group of patients (Fig. 1A). Also, we detected the expression of miR-409-3p in a normal human bronchial epithelial cell line (HBE) and three LAD cell lines (A549, SPC-A1 and H1299), and showed that the relative expression level of miR-409-3p in LAD cell lines was significantly lower than that in normal human bronchial epithelial cell line (Fig. 1B). By statistical analyses, it was shown that the miR-409-3p expression levels were significantly lower in the LAD tissues than the matched adjacent non-cancerous tissues $(P=0.013$; Fig. 1C). Also, we detected the expression of miR-409-3p in tumors with various pTNM stage, and results showed that miR-409-3p was expressed at relatively high levels in tumors with an early pTNM stage (pI and pII), and significantly downregulated in pIII tumors and further downregulated in pIV tumors (Fig. 1D). Also, the expression of miR-409-3p in LAD tissues with or without lymph node metastasis was analyzed. Results indicated that the miR-409-3p expression levels were significantly lower in tumors with lymph node metastasis than those without lymph node metastasis $(P=0.007$; Fig. $1 \mathrm{E})$. Thus, these data indicated that lower miR-409-3p expression might be involved in LAD development. 
Fig. 1. The expression levels of miR-409-3p in LAD tissues. (A) qRTPCR assay was performed to detect the relative expression of mature miR-409-3p in 34 LAD and corresponding non-cancerous tissues. (B) qRT-PCR detection of miR-409$3 p$ expression in three LAD cell lines (A549, SPC-A1 and PC9). U6 was used as an internal control. (C) Calculating the mean level of miR-409-3p in 34 cases of LAD tissues (T) and corresponding noncancerous tissues (N), respectively $(P=0.013)$. (D) Detection of miR-409-3p expression in tumor tissues with various pTNM stage (pI, pII, pIII and pIV). (E) Detection of miR-409-3p

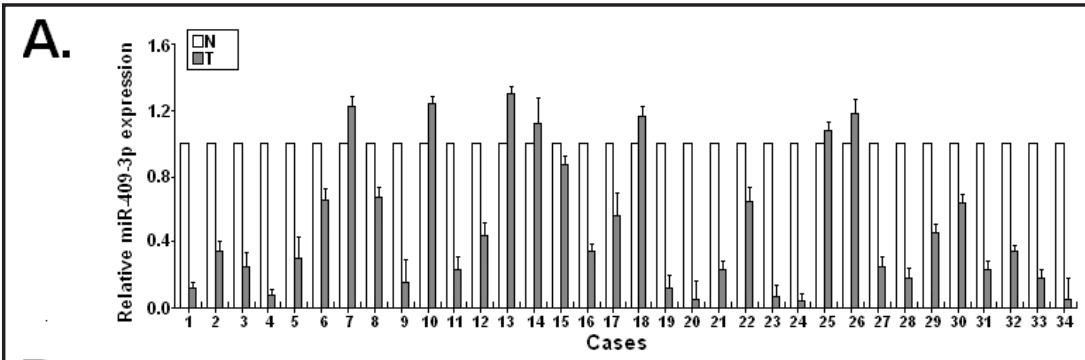

B.

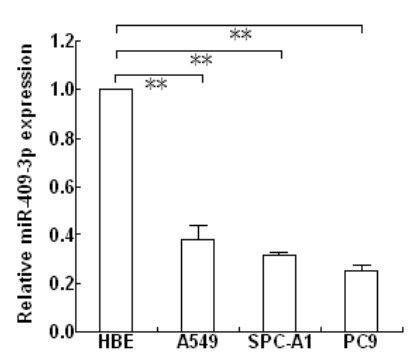

D.

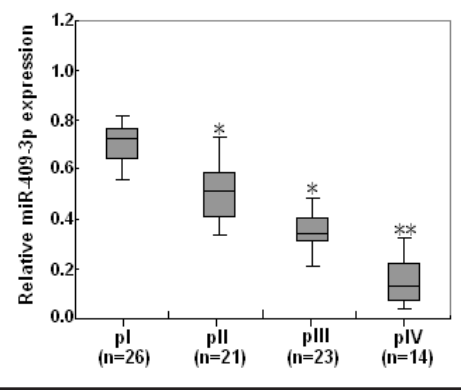

C.

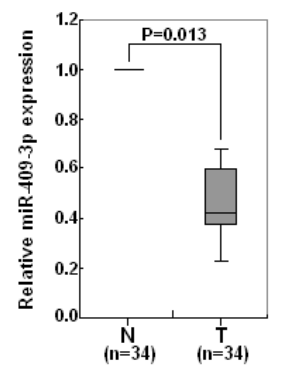

E.

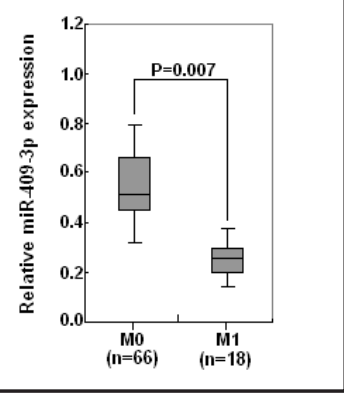

expression in metastatic and nonmetastatic LAD tissues. The mean and standard deviation of expression levels relative to U6 expression levels are shown and are normalized to the expression in the normal tissue of each matched pair. Each experiment was performed at least in triplicate. ${ }^{*} P<0.05,{ }^{*} P<0.01$.

Table 2. Univariate and multivariate analysis of prognostic factors in LAD for 5-year OS. ${ }^{*} P<0.05$. Abbreviations: HR, hazard ratio; $95 \% \mathrm{CI}, 95 \%$ confidence interval; OS, overall survival. TNM, lymph-node-metastasis and stage according to the TNM classification for lung cancer (UICC)

\begin{tabular}{|c|c|c|c|c|}
\hline \multirow[b]{2}{*}{ Clinicopathological factors } & \multicolumn{2}{|l|}{ Univariate analysis } & \multicolumn{2}{|c|}{ Multivariate analysis } \\
\hline & HR $(95 \%$ CI $)$ & $P$-value & $\operatorname{HR}(95 \% \mathrm{CI})$ & $P$-value \\
\hline Age $(\geq 60$ vs $<60$ years $)$ & $1.88(0.91-2.34)$ & 0.268 & - & - \\
\hline Gender (Male vs Female) & $2.11(0.87-2.48)$ & 0.167 & - & - \\
\hline Smoking (Smoker vs non-smoker) & $1.23(0.69-1.88)$ & 0.312 & - & - \\
\hline $\begin{array}{l}\text { Tumor differentiation } \\
\text { (Poor+Moderate vs Well) }\end{array}$ & $1.37(0.71-1.65)$ & $0.008^{*}$ & $1.68(0.83-2.49)$ & 0.378 \\
\hline pTNM stage (Stage III/IV vs Stage I/II) & $2.78(1.44-3.02$ & $0.016^{*}$ & $1.45(1.03-2.22)$ & $0.026^{*}$ \\
\hline miR-409-3p expression (Low vs High) & $1.76(1.27-3.13)$ & $0.006^{*}$ & $1.83(1.44-2.28)$ & $0.038^{*}$ \\
\hline
\end{tabular}

Correlation of miR-409-3p expression with clinicopathologic factors of LAD patients

To further investigate the correlation between miR-409-3p expression and clinicopathologic factors of LAD patient, the levels of miR-409-3p were quantified in 


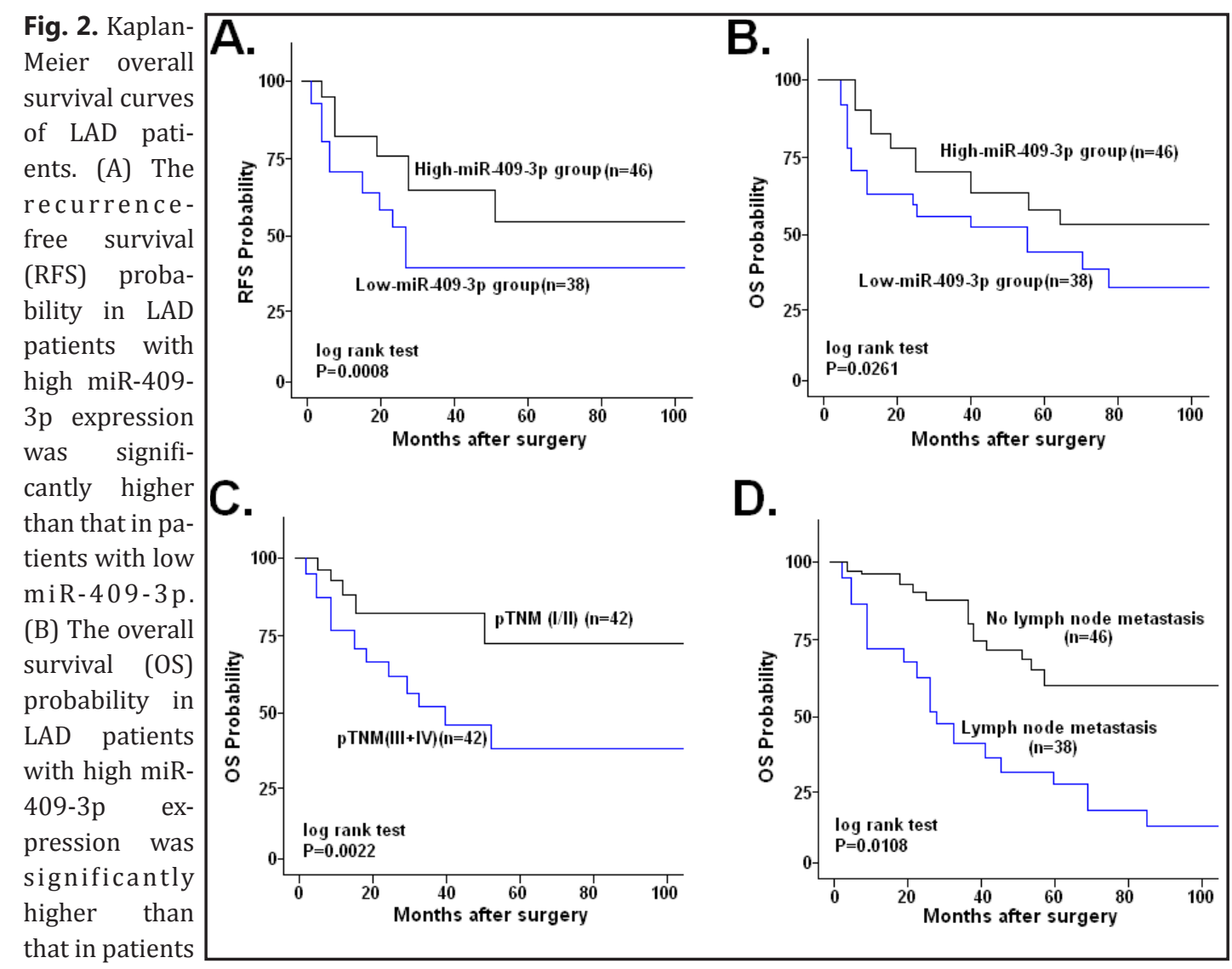

with low miR-409-3p expression. (C) The OS probability in LAD patients with high pTNM stage (pIII+IV) was significantly lower than that in patients with low pTNM stage (pI+II). (D) The OS probability in LAD patients with lymph node metastasis was significantly lower than that in patients without lymph node metastasis. ${ }^{*} P<0.05,{ }^{* *} P<0.01$.

another cohort of 84 LAD tissue samples using real-time RT-PCR. After normalization to U6 expression levels, the expression level of miR-409-3p in LAD tissues (mean \pm SD: $0.236 \pm 0.15$ ) was significantly lower than that in adjacent normal tissues (mean \pm SD:1.353 \pm 0.46 , $P<0.001)$. The median expression level of miR-409-3p (0.236) was used as a cutoff point to divide all 84 patients into two groups: LAD patients who express miR-409-3p at levels less than the cutoff value were assigned to the low expression group (mean expression value $0.158, \mathrm{n}=38$ ), and those with expression above the cutoff value were assigned to the high expression group (mean expression value $0.697, \mathrm{n}=46$ ). As shown in Table. 1, statistical analyses indicated that low miR-409-3p expression in LAD was significantly correlated with poor tumor differentiation, advanced pTNM stage and higher incidence of lymph node metastasis $(P=0.032,0.028$ and 0.020 , respectively). However, there were no statistically significant correlation between miR-409-3p expression and other factors including age, gender, smoking condition, T-primary tumor and cancer-related death $(P=0.744,0.433$, $0.071,0.569$ and 0.411 , respectively).

\section{MiR-409-3p is an independent prognostic factor for LAD patients}

Next, we investigated the correlation of miR-409-3p expression with prognosis of LAD patients. Kaplan-Meier survival curves were plotted according to miR-409-3p expression, pTNM stage and status of lymph node metastasis. As shown in Fig. 2A, LAD patients with low-miR-409-3p expression had a lower recurrence-free survival than those patients with high-miR-409-3p expression $(P=0.0008)$. As shown in Fig. 2B, LAD patients with high-miR409-3p expression showed a better prognosis than those with low-miR-409-3p expression 


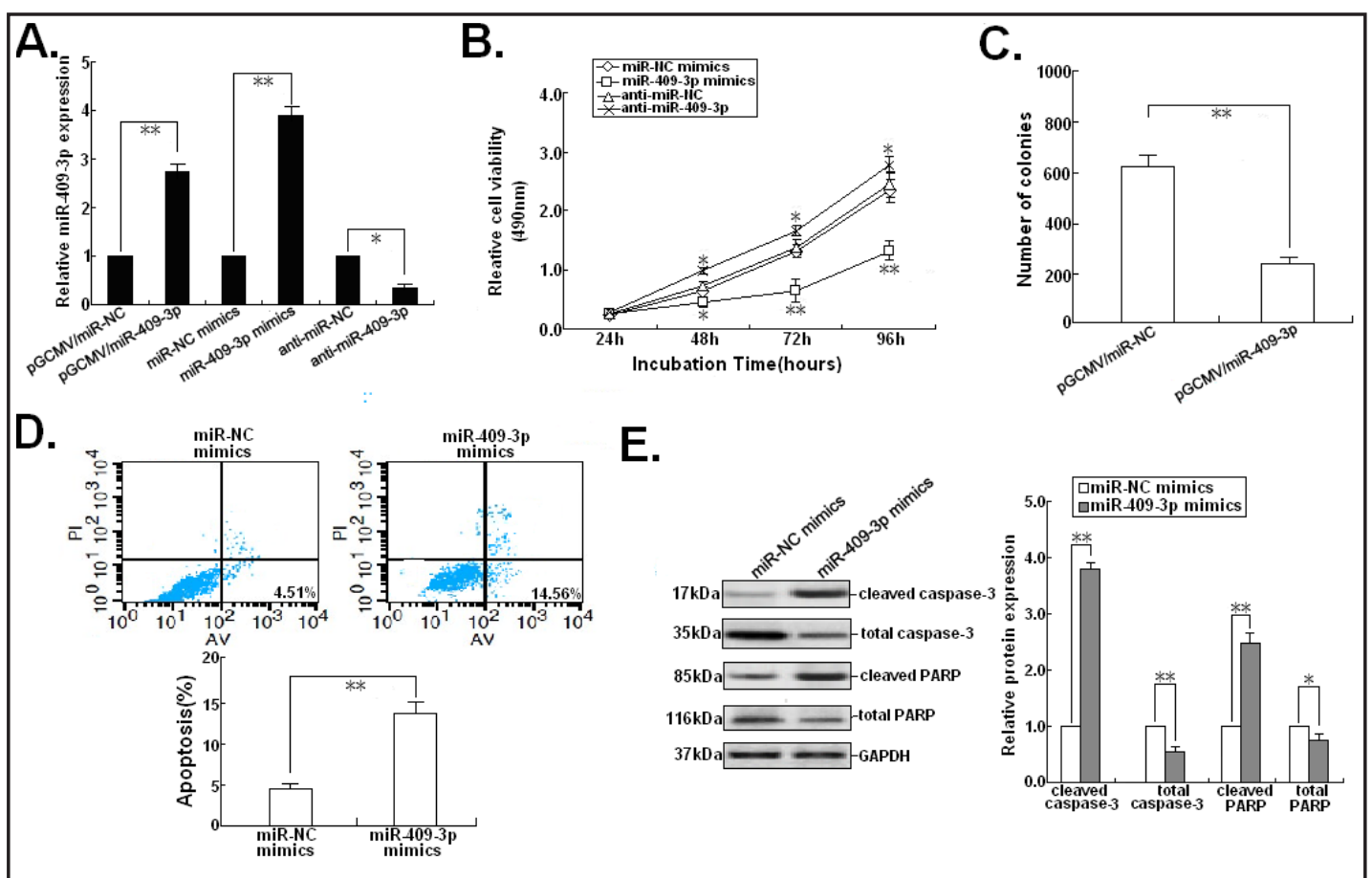

Fig. 3. Effects of miR-409-3p expression on growth, colony formation and apoptosis of A549 cells. (A) 48h after transient transfection with miR-409-3p mimics (or miR-NC mimics) or anti-miR-409-3p (or anti-miR$\mathrm{NC}$ ) or stable transfection with $\mathrm{pGCMV} / \mathrm{miR}-409-3 \mathrm{p}$ or $\mathrm{pGCMV} / \mathrm{miR}-\mathrm{NC}$, qRT-PCR assay was performed to detect the expression of miR-409-3p. U6 was used as an internal control. (B) MTT analysis of growth in A549 cells at different time points after transfection. (C) The colony formation assay was performed as described in Methods using A549 cells stably transfected with pGCMV/miR-409-3p or pGCMV/miR-NC. The number of colonies was counted and compared. (D) The apoptosis of A549 cells transfected with miR-409$3 p$ mimics or control mimics was determined by flow cytometry. (E) Western blot analysis of the expression levels of cleaved caspase-3, total caspase-3, cleaved PARP and total PARP proteins. GAPDH was used as an internal control. Each experiment was performed at least in triplicate. ${ }^{*} P<0.05,{ }^{* *} P<0.01$ versus control. N.S: not significance versus control.

$(P=0.0261)$. The median survival time for the LAD patients with high-miR-409-3p was 52.4 months, and for LAD patients with low-miR-409-3p was 32.4 months. Also, we also found that patients with pTNM stage (pIII+IV) had shorter survival time than those with pTNM stage (pI+II) ( $P=0.0022$; Fig. 2C). Additionally, LAD patients with lymph node metastasis had a shorter survival time than those with no lymph node metastasis $(P=0.0108$; Fig. 2D). The data of univariate and multivariate analyses of factors correlated with prognosis of LAD patient were indicated in Table. 2. By univariate survival analysis, it was observed that tumor differentiation, pTNM stage, and miR-409-3p expression were closely correlated with 5 -year OS of LAD patients $(P=0.008,0.016$ and 0.006 , respectively). Further, multivariate Cox regression analyses showed that status of miR-409-3p, along with pTNM stage, was independent prognostic factors for LAD patients $(P=0.038$ and 0.026 , respectively).

MiR-409-3p inhibits growth and enhances apoptosis in LAD cells

To better understanding the biological functions of miR-409-3p in the development of LAD, we first investigate the effect of miR-409-3p on growth and apoptosis of LAD cells. 48h After LAD cell line (A549) was transfected with miR-409-3p mimics or miR-409-3p inhibitor (anti-miR-409-3p), qRT-PCR was performed to detect the expression of miR-4093p. As shown in Fig. 3A, compared with control mimics or anti-miR-NC-transfected cells, the expression of miR-409-3p was significantly increased in miR-409-3p mimics-transfected 


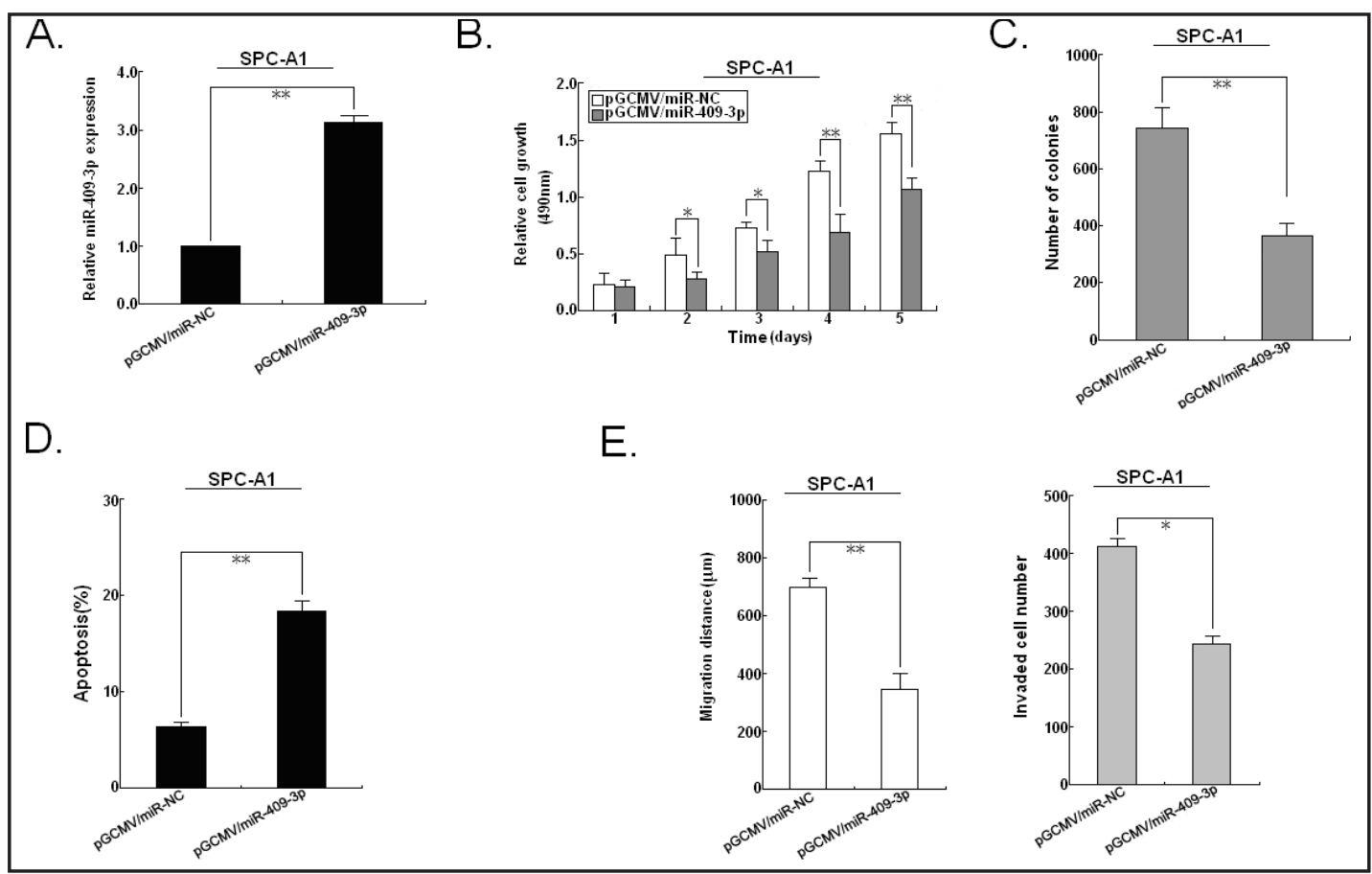

Fig. 4. Effects of miR-409-3p expression on growth, apoptosis, migration and invasion in LAD cell line (SPCA1). (A) qRT-PCR detection of miR-409-3p expression in SPC-A1 cells stably transfected with pGCMV/miR409-3p or pGCMV/miR-NC, respectively. U6 was used as an internal control. (B) MTT analysis of growth in stably transfected SPC-A1 cells at different time points. (C) The colony formation assay was performed as described in Methods using SPC-A1 cells stably transfected with pGCMV/miR-409-3p or pGCMV/miR-NC. The number of colonies was counted and compared. (D) The apoptosis of SPC-A1 cells stably transfected with pGCMV/miR-409-3p or pGCMV/miR-NC was detected by flow cytometry. (E) Wound healing Transwell invasion assays were performed to analyze the migration and invasion of SPC-A1 cells stably transfected with pGCMV/miR-409-3p or pGCMV/miR-NC. Results represent the average of three independent experiments (mean $\pm \mathrm{SD}$ ). ${ }^{*} P<0.05,{ }^{* *} P<0.01$ versus control. N.S: not significance versus control.

A549 cells $(P<0.01)$ and was significantly downregulated in anti-miR-409-3p-transfected cells $(P<0.05)$. First, MTT assay indicated that transfection of miR-409-3p mimics could significantly inhibit growth but downregulation of miR-409-3p could moderately increase growth in A549 cells (Fig. 3B). Colony formation assay showed that the ability of colony formation in A549 cells stably transfected with pGCMV/miR-409-3p was significantly reduced in comparison with that in miR-NC mimics-transfected cells $(P<0.01 ;$ Fig. 3C). In addition, transfection of miR-409-3p mimics induced apoptosis $(14.56 \%$ versus $4.51 \%$ in the control group; $P<0.01$; Fig. 3D). Furthermore, the expression of cleaved caspase- 3 and PARP proteins was significantly upregulated in miR-409-3p mimics-transfected A549 cells compared with miR-NC mimics-transfected cells (Fig. 3E). Likewise, we also confirmed that upregulation of miR-409-3p could inhibit growth and increase apoptosis in another LAD cell line (SPC-A1) (Fig. 4A-D). Collectively, miR-409-3p significantly inhibits the growth of LAD cells by promoting caspase-3-dependent apoptosis.

\section{MiR-409-3p inhibits in vitro migration and invasion in LAD cells}

Then, we will investigate the effect of miR-409-3p expression on migration and invasion of LAD cells. Wound healing assays showed that the migration of A549 cells was significantly decreased after transfection of miR-409-3p mimics (Fig. 5A). In contrast, anti-miR-409-3p increased wound healing (Fig. 5B). Similarly, in Matrigel invasion assays, upregulation of miR-409-3p could significantly reduce invasion of A549 cells (123 versus $412, P<0.01$; Fig. 5C). Likewise, downregulation of miR-409-3p could markedly increase invasion of A549 cells 
Fig. 5. Effects of miR-409$3 p$ expression on migration and invasion in A549 cells. Wound healing assay was performed to analyze the effects of miR-409-3p upregulation (A) or downregulation (B) on migration of A549 cells as described in Methods. Transwell invasion assay was performed to analyze the effect of miR-409-3p upregulation (C) or down-

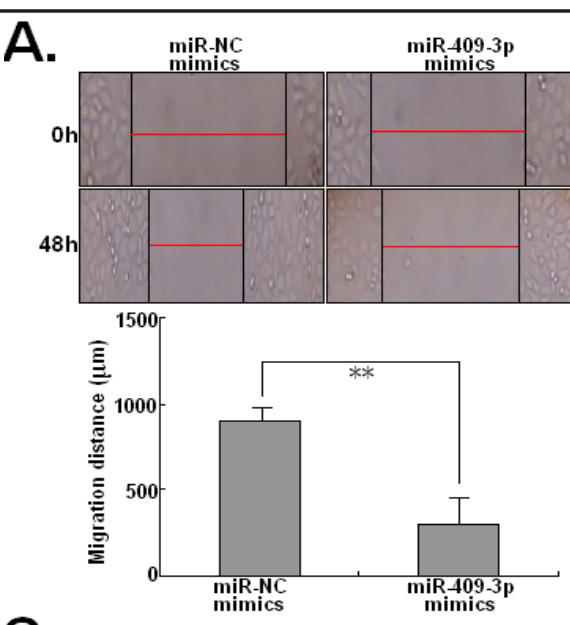

B.

C.

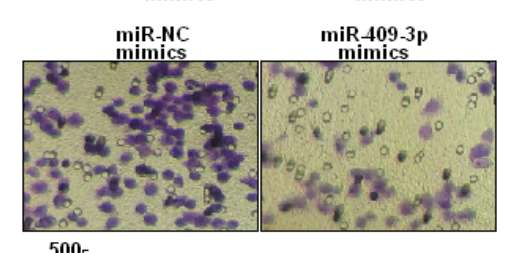

D.
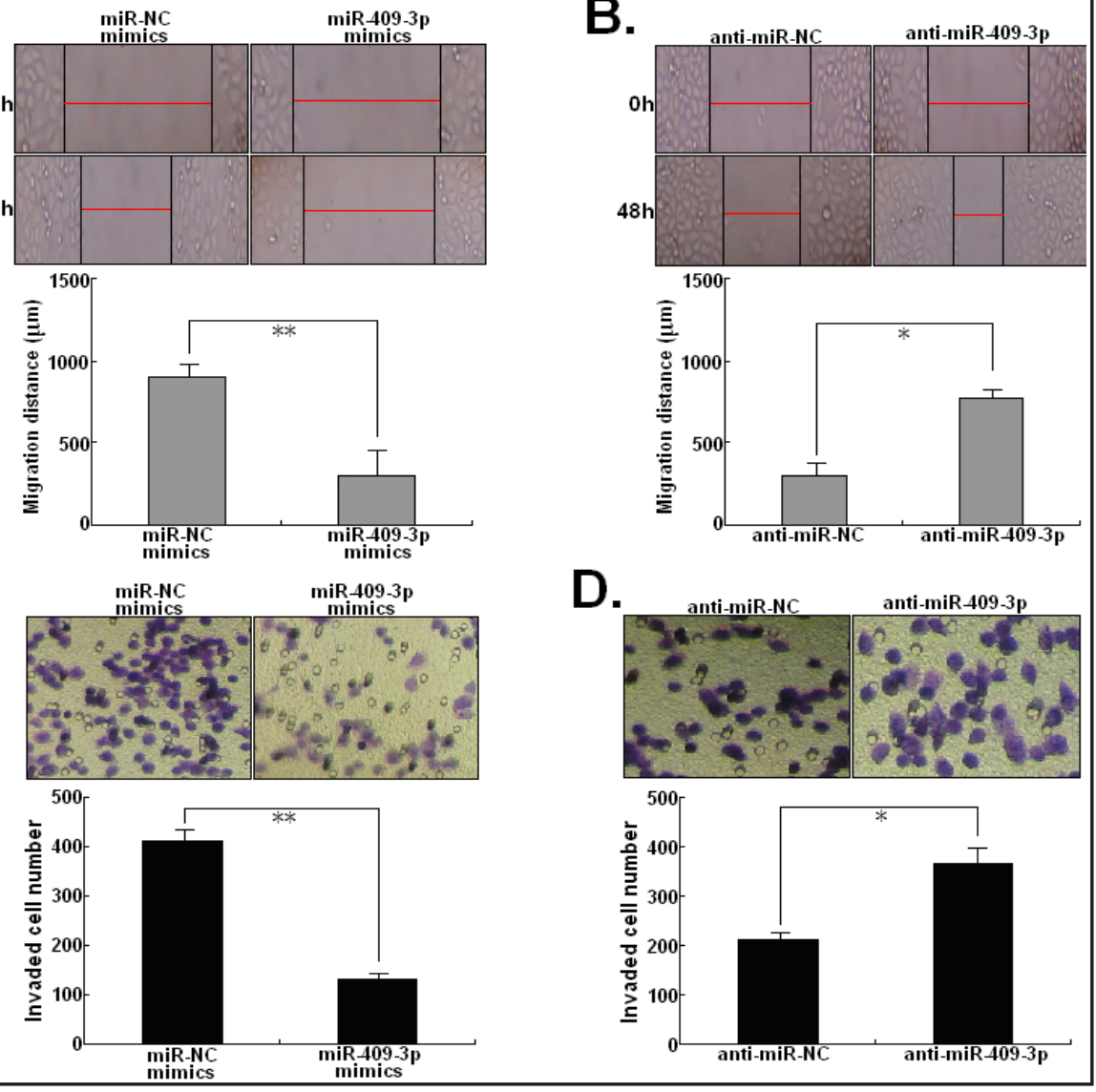

regulation (D) on invasion of A549 cells. Each experiment was performed at least in triplicate. ${ }^{*} P<0.05$, ${ }^{* *} P<0.01$ versus control.

(206 versus 354, $P<0.05$; Fig. 5D). Likewise, we also confirmed that stable transfection of miR-409-3p could reduce migration and invasion in SPC-A1 cell line (Fig. 4E). These results suggest that miR-409-3p significantly inhibits in vitro migration and invasion of LAD cells.

\section{c-Met is identified as a direct target of miR-409-3p}

To investigate the targets of miR-409-3p to elucidate the underlying mechanisms of its effects, we performed miRNA target gene prediction with PicTar, TargetScan and Miranda databases. The c-Met exhibited miR-409-3p-binding sequences in its 3'-UTR regions (Fig. 6A), and in silico analysis showed 3'-UTR of human c-Met (4912-4978 nt) contains a potential miR-409-3p binding site. To further confirm that the c-Met 3'-UTR is a target for miR-409-3p, we subcloned the fragment of c-Met 3'-UTR harboring the potential binding site into downstream of the pEZX-Luc vector to generate the pEZX-luc-c-Met/3'-UTR-wt vector and mutated the miR-409-3p binding site on c-Met 3'-UTR (c-Met/3'-UTR-mut) and subcloned it into pEZX-luc vector generate the pEZX-luc-c-Met/3'-UTR-mut vector. Those vectors were co-transfected into A549 cells with miR-409-3p mimics (or miR-NC mimics) or anti-miR-409-3p (or anti-miR-NC), and luciferase activity was determined. As shown in Fig. 6B, the luciferase activity was decreased by miR-409-3p mimics $(P<0.01)$ when the wildtype c-Met/3'-UTR was present, and the activity was increased $(P<0.05)$ when miR-409$3 p$ was downregulated. However, the mutations of c-Met/3'-UTR prevented the expression of miR-409-3p from affecting luciferase activity. Next, we analyzed the effect of miR-409$3 p$ expression on the expression of c-Met in LAD cells. As shown in Fig. 6C, transfection of miR-409-3p mimics could lead to the decreased expression of c-Met protein in A549 cells $(P<0.05)$, while transfection of miR-409-3p inhibitor could lead to the increased expression 


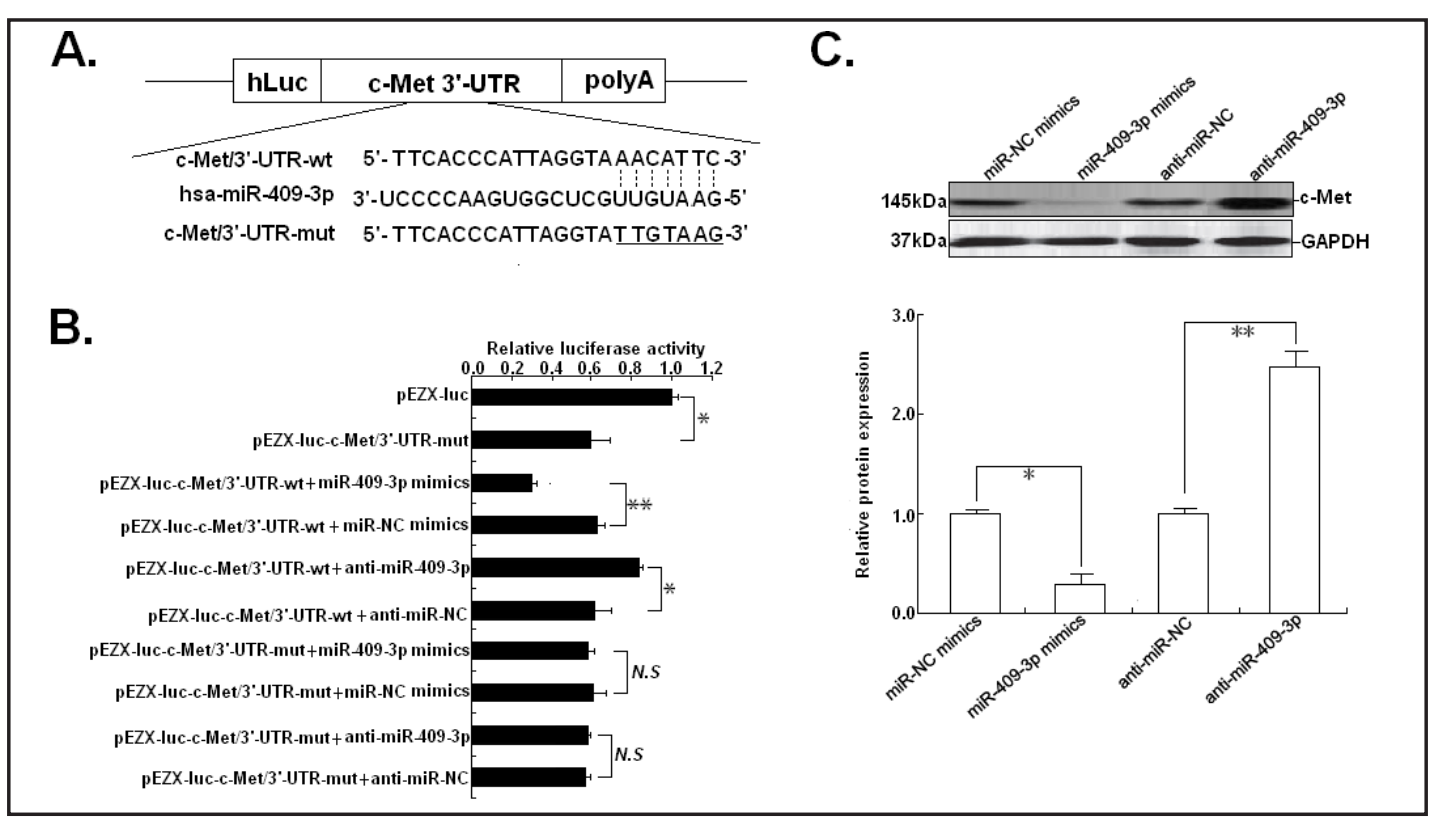

Fig. 6. MiR-409-3p binds to the 3'-UTR of c-Met mRNA. (A) miR-409-3p and its putative binding sequence in the 3'-UTR of c-Met. The mutant miR-409-3p-binding site wa generated in the complementary site for the seed region of miR-409-3p. (B) Relative luciferase activity was analyzed. pEZX-luc-c-Met/3'-UTR-wt or pEZX-luc-c-Met/3'-UTR-mut vector and miR-409-3p mimics (or control mimics) or anti-miR-409-3p (or anti-miR-NC) were co-transfected into A549 cells, Cells lysates were prepared after $48 \mathrm{~h}$ for measuring luciferase activity, which was normalized to normalized to Renilla luciferase activity. The histogram shows the mean \pm SD of the normalized luciferase activity from three independent experiments. (C) Western blotting detection of c-Met protein expression in A549 cells transfected with miR-409-3p mimics (or miR-NC mimics) or anti-miR-409-3p (or anti-miR-NC). GAPDH was used as an internal control. Data were presented as mean $\pm \operatorname{SEM}(\mathrm{n}=3) .{ }^{*} P<0.05,{ }^{* *} P<0.01$ versus control. $N . S$ : not significance versus control.

of c-Met protein $(P<0.05)$. These data indicate that c-Met is a direct target of miR-409-3p in LAD.

shRNA-mediated c-Met downregulation inhibits growth, induce apoptosis, reduce migration and invasion in LAD cells

Previous studies have shown that c-Met (hepatocyte growth factor receptor), a tyrosine kinase receptor for hepatocyte growth factor (HGF), plays an important role in malignant transformation and tumor development by activating mitogenic signaling pathways $[16,17]$. To better understand the biological functions of c-Met in LAD, shRNA/c-Met1 or 2 and shRNA/ control vector was stably transfected into A549 cells, respectively. qRT-PCR and Western blot assays confirmed the downregulation of c-Met mRNA and protein induced by shRNA/c-Met1 or shRNA/c-Met2, and shRNA/c-Met2 exhibited the stronger inhibitory effect (Fig. 7A). Thus, shRNA/c-Met2 was chosen for further functional analyses. Results from MTT assay indicated that siRNA-mediated downregulation of c-Met could significantly inhibit growth of A549 cells and the highest inhibitory rate was $36.6 \pm 2.3 \%$ at 5 days $(P<0.01 ;$ Fig. $7 \mathrm{~B})$. Compared with shRNA/control-transfected cells, the apoptotic rate of shRNA/c-Met2-transfected A549 cells was significantly increased by about $22.4 \%(P<0.01$; Fig. 7 C). Meanwhile, silencing of c-Met2 significantly increased the expression of cleaved caspase- 3 and PARP proteins in A549 cells (Fig. 7D). Wound healing and Matrigel invasion assays indicated that shRNA/cMet 2 could significantly reduce the capacities of migration and invasion in A549 cells (Fig. $7 \mathrm{E}$ and F). These results suggest that shRNA-mediated downregulation of c-Met can mimic the effect of miR-409-3p upregulation on phenotypes of LAD cells. 


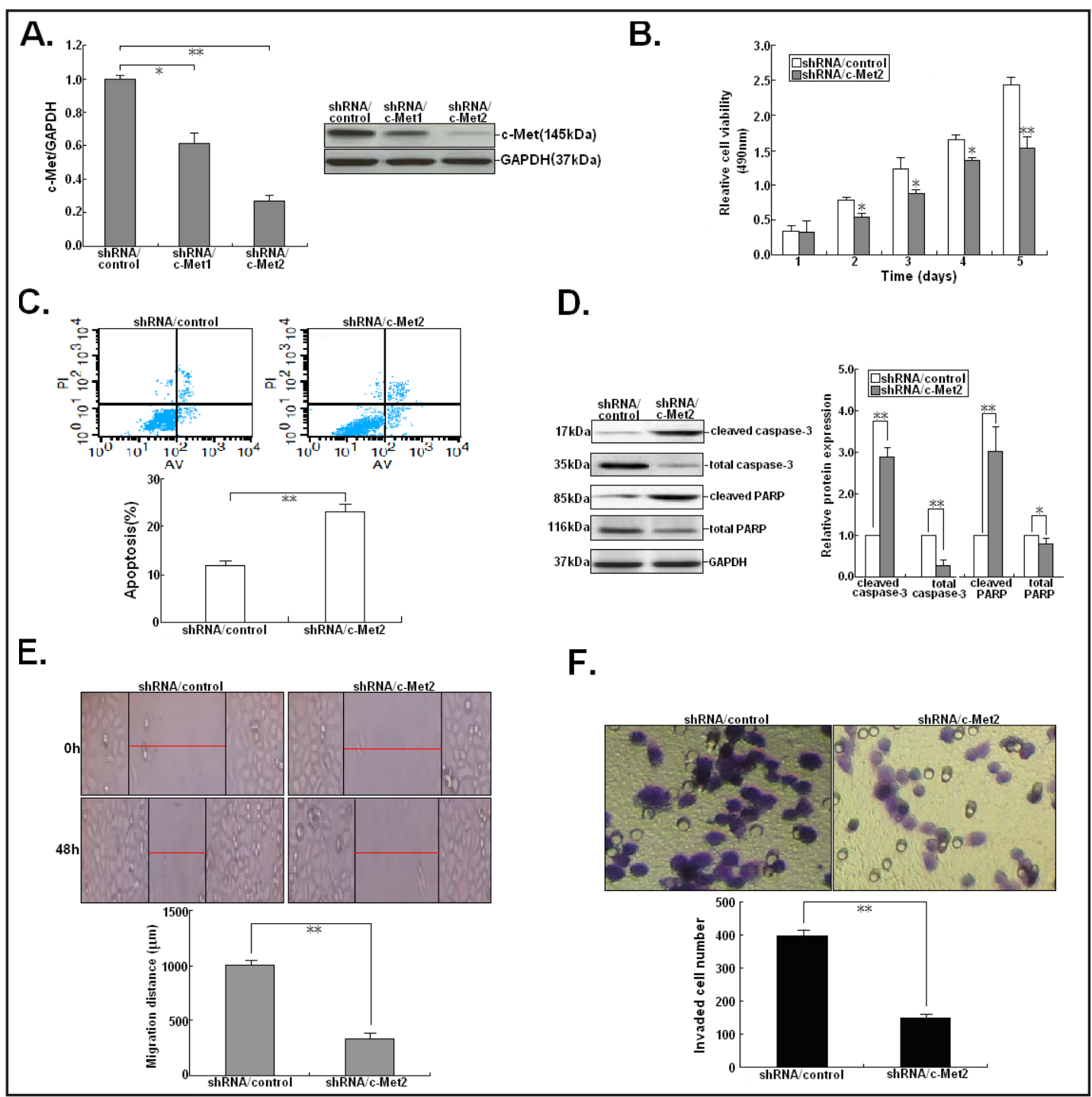

Fig. 7. Effects of c-Met downregulation on growth, apoptosis, migration and invasion in A549 cells. (A) qRTPCR and Western blotting assays were performed to detect the expression of c-Met mRNA and protein in A549 cells stably transfected with shRNA/control, shRNA/c-Met1 or shRNA/c-Met2, respectively. (B) MTT analysis of growth in A549 cells stably transfected with shRNA/control or shRNA/c-Met2 at different time points. (C) Flow cytometry detection of apoptosis in A549 cells stably transfected with shRNA/control or shRNA/c-Met2, respectively. (D) Western blot analysis of the expression levels of cleaved caspase-3, total caspase-3, cleaved PARP and total PARP proteins in A549 cells stably transfected with shRNA/control or shRNA/c-Met2, respectively. GAPDH was used as an internal control. (E) Wound healing assay was performed to analyze the effects of c-Met downregulation on migration of A549 cells. (F) Transwell invasion assay was performed to analyze the effect of c-Met downregulation on invasion of A549 cells. Each experiment was performed at least in triplicate. ${ }^{*} P<0.05$, ${ }^{* *} P<0.01$ versus control. N.S: not significance versus control.

Upregulation of c-Met partially rescues the effects of miR-409-3p on growth, apoptosis, migration and invasion in LAD cells

To further investigate the roles of c-Met in phenotypical changes of LAD cells induced by miR-409-3p, A549 cells were co-transfected with miR-409-3p mimics (or control mimics) and pcDNA/c-Met vector. $48 \mathrm{~h}$ after co-tranfection, Western blotting assay was performed to detect the expression of c-Met protein. As shown in Fig. 8A, the co-transfection could rescue the decreased expression of c-Met protein in A549 cells induced by miR-409-3p mimics. 


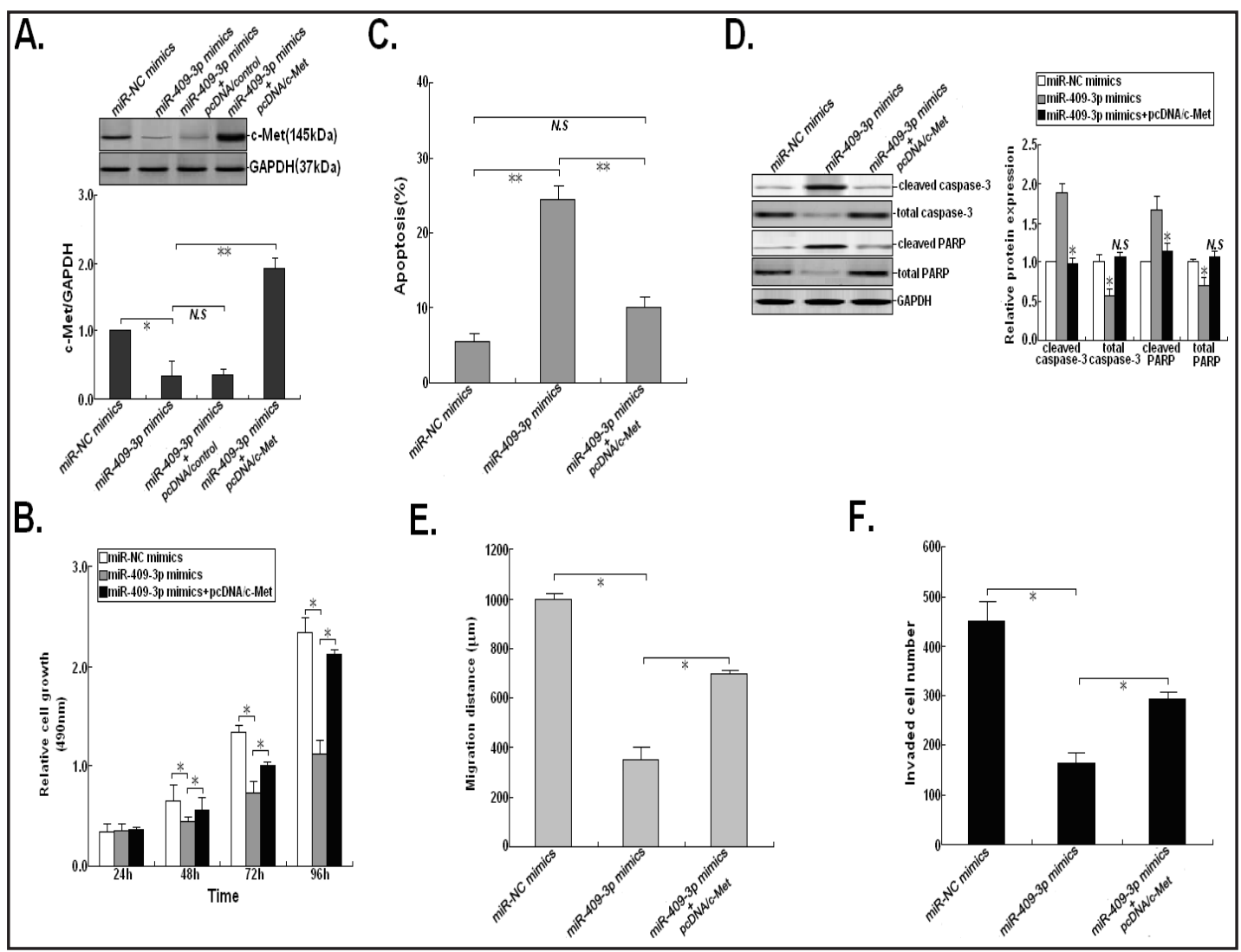

Fig. 8. Overexpression of c-Met partially rescues the effects of miR-409-3p upregulation on phenotypes of A549 cells. (A) 48h after A549 cells were transfected with miR-409-3p mimics (or miR-NC mimics) or cotransfected with miR-409-3p mimics (or miR-NC mimics) and shRNA/c-Met2 (or shRNA/control) vector, Western blotting assay was performed to detect the expression of c-Met protein. (B) MTT analysis of growth in A549 cells transfected with miR-409-3p mimics (or control mimics) or co-transfected with miR-409-3p mimics and shRNA/c-Met2 vector. (C) Flow cytometry assay was performed to detect apoptosis of A549 cells transfected with miR-409-3p mimics (or miR-NC mimics) or co-transfected with miR-409-3p mimics and shRNA/c-Met2 vector. (D) Western blot analysis of the expression levels of cleaved caspase-3, total caspase-3, cleaved PARP and total PARP proteins in A549 cells transfected with miR-409-3p mimics (or miR-NC mimics) or co-transfected with miR-409-3p mimics and shRNA/c-Met2 vector. (E) Wound healing assay was performed to analyze the migration of A549 cells transfected with miR-409-3p mimics (or miR-NC mimics) or co-transfected with miR-409-3p mimics and shRNA/c-Met2 vector. (F) Transwell invasion assay was performed to analyzed the invasion of A549 cells transfected with miR-409-3p mimics (or control mimics) or co-transfected with miR-409-3p mimics and shRNA/c-Met2 vector. GAPDH was used as an internal control. Each experiment was performed at least in triplicate. ${ }^{*} P<0.05,{ }^{* *} P<0.01$ versus control. $N . S$ : not significance versus control.

Meanwhile, we found that overexpression of c-Met could reverse the inhibition of growth in A549 cells induced by miR-409-3p mimics (Fig. 8B). The enhancement of apoptosis and the increased expression of cleaved caspase- 3 or PARP proteins in A549 cells induced by miR409-3p mimics could also be partially reversed by c-Met upregulation (Fig. 8C and D). In addition, overexpression of c-Met could reverse the inhibitory effect of miR-409-3p mimics on the activities of migration and invasion in A549 cells (Fig. 8E and F). Therefore, these findings further indicate that c-Met is a functional target of miR-409-3p in LAD cells.

Akt signaling mediates the downstream effects of miR-409-3p/c-Met in LAD cells

Previously, it has been reported that aberrant hepatocyte growth factor/Met signaling induces centrosome amplification via the Akt signaling pathway [18], we then explore 
Fig. 9. Effects of miR-409$3 \mathrm{p}$ mimics and shRNA/cMet2 on the expression of Akt signalingrelated proteins in A549 cells. (A) Western blotting detection of $p$ c-Met protein expression in miR-409$3 p$ mimics or shRNA/cMet2 - transfected cells. (B) Western blotting detection of the expression of pAkt and total Akt proteins in miR-409$3 p$ mimics or shRNA/cMet2-transfected cells. (C) Western blotting detection of the expression of Bcl-2 and Bax
A.

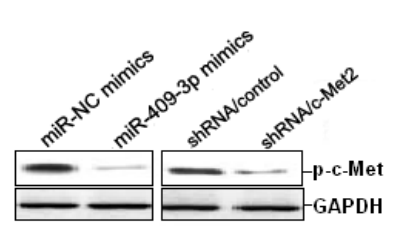

B.
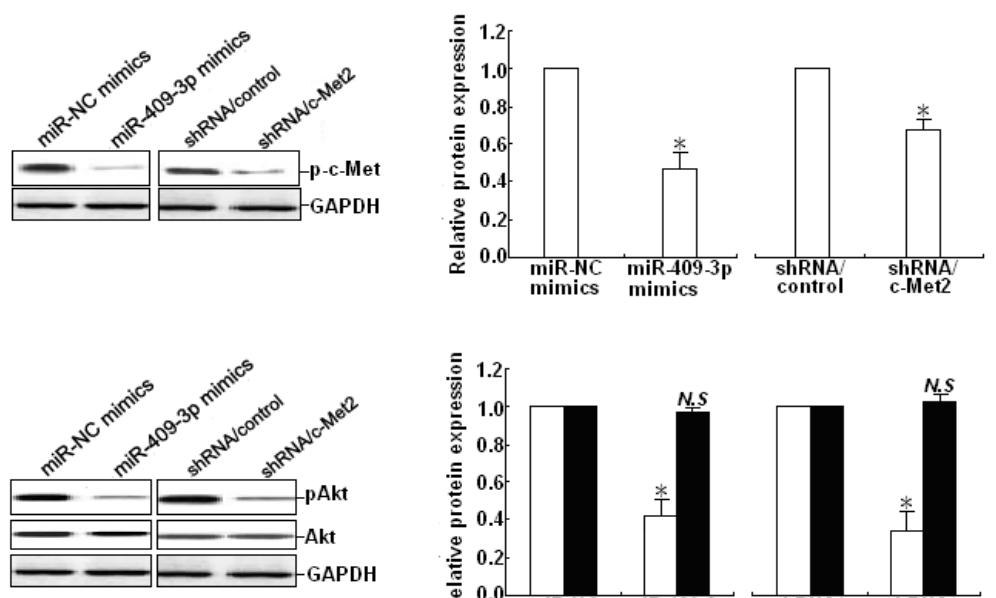

ㅁp-c-Met

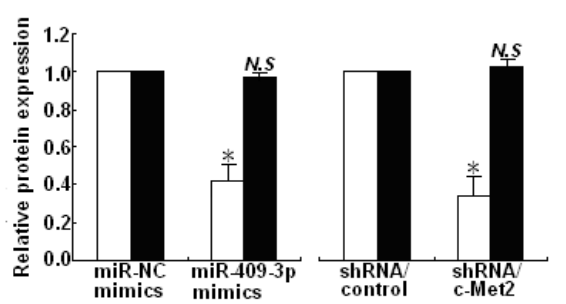

qpAkt

C.
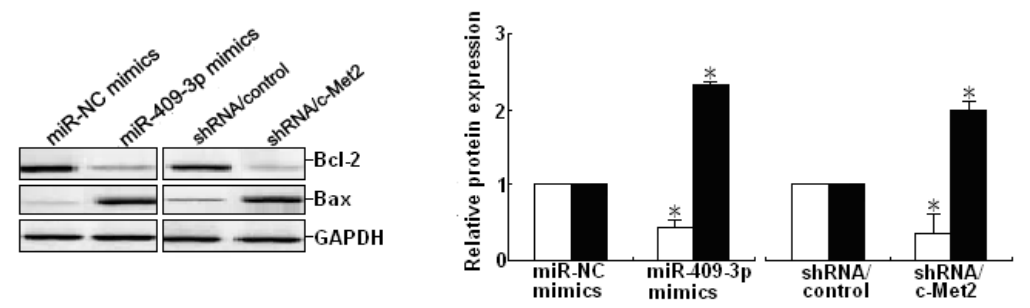

$\square$ Bcl-2

D.

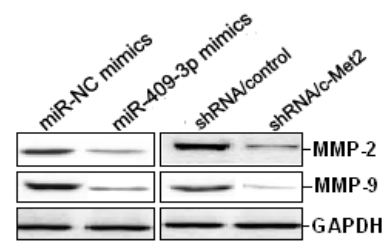

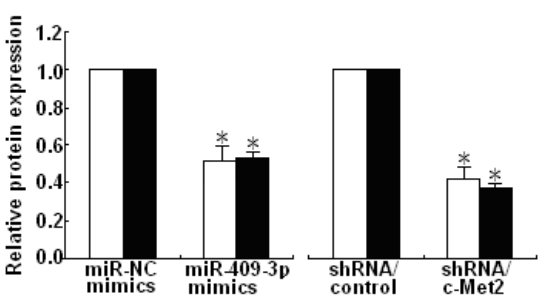

DMMP -MMP_-9

proteins in miR-409-3p mimics or shRNA/c-Met2-transfected cells. (D) Western blotting detection of the expression of MMP-2 and MMP-9 proteins in miR-409-3p mimics or shRNA/c-Met2-transfected cells. GAPDH was used as an internal control. Each experiment was performed at least in triplicate. ${ }^{*} P<0.05$, ${ }^{* *} P<0.01$ versus control. N.S: not significance versus control.

whether miR-409-3p and c-Met mediated the regulation of malignant phenotypes of LAD cells by affecting the Akt signaling. First, we analyzed the effect of miR-409-3p and c-Met expression on phosphorylated c-Met (p-c-Met) in LAD cells, and showed that upregulation of miR-409-3p and downregulation of c-Met significantly inhibit p-c-Met protein expression in LAD cells (Fig. 9A). Also, both upregulation of miR-409-3p and downregulation of c-Met induced the inhibition of phosphorylated Akt (pAkt) protein expression but had no effects on the expression of total Akt protein in A549 cells (Fig. 9B). Then, we detect the expression of downstream targets of Akt signaling, including Bcl-2, Bax, MMP-2 and MMP-9. Likewise, we showed that both miRNA-409-3p mimics and shRNA/c-Met2 could inhibit the expression of $\mathrm{Bcl}-2$ protein and increase the expression of Bax protein, which induced the decreased ratio of Bcl-2/Bax (Fig. 9C). Also, the expression of MMP-2 and MMP-9 proteins was observed to be significantly downregulated in both miR-409-3p mimics and shRNA/c-Met2-transfected cells (Fig. 9D). Those targets of Akt signaling was correlated with growth and metastasis of LAD cells, so miR-409-3p might affect phenotypes of LAD cells via regulation of those 


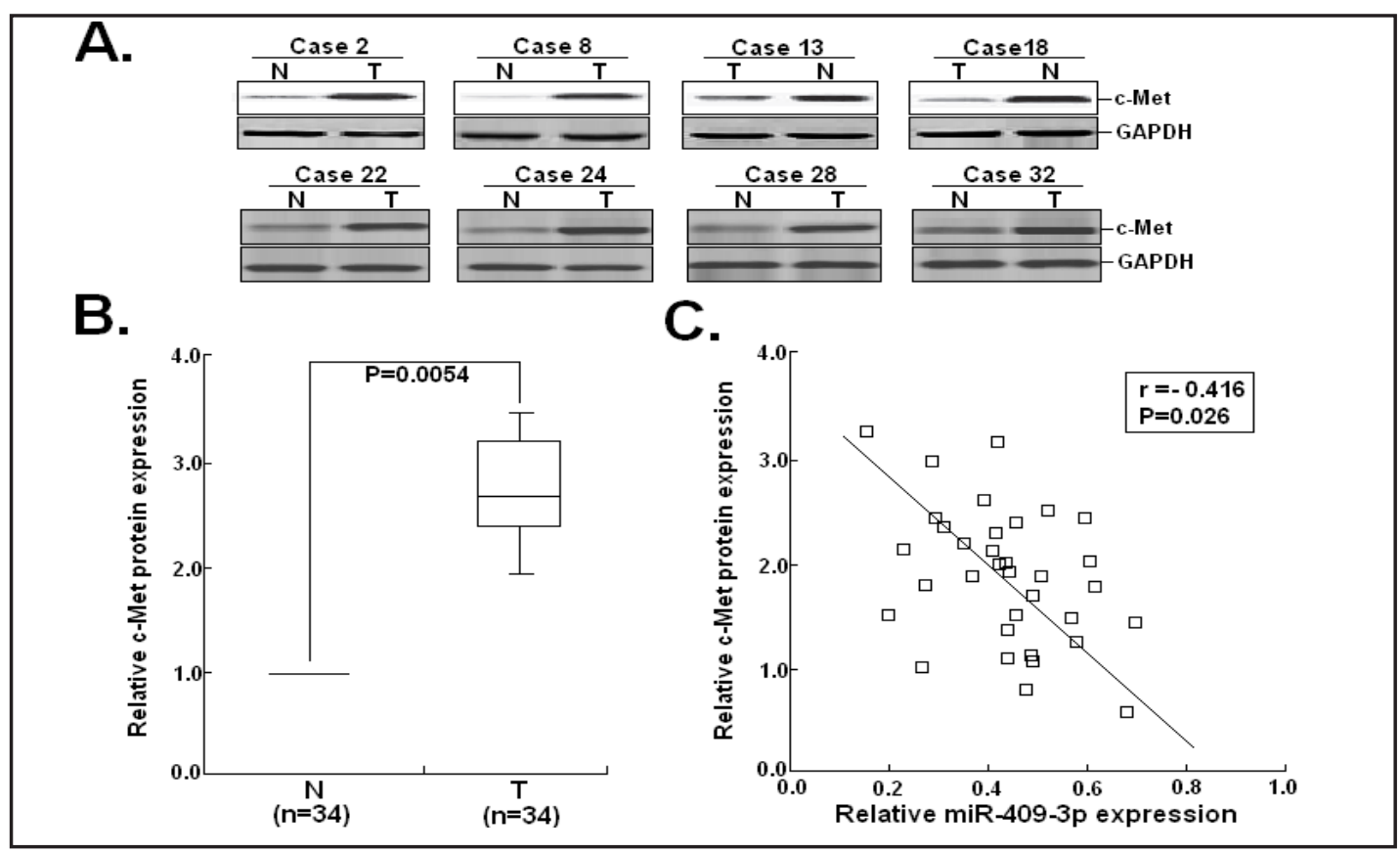

Fig. 10. The expression level of c-Met protein was significantly downregulated and inversely correlated with miR-409-3p expression level in LAD tissues. (A) Western blotting detection of c-Met protein expression in 34 cases of LAD and corresponding non-cancerous tissues. GAPDH was used as an internal control. (B) Calculating the mean level of c-Met protein in 34 cases of LAD tissues ( $\mathrm{T}$ ) and corresponding non-cancerous tissues $(N)$, respectively $(P=0.0054)$. (C) A statistically significant inverse correlation between miR-409-3p and c-Met protein expression levels in 34 cases of LAD tissues (Spearman's correlation analysis, $r=-0.416$; $P=0.026$ ). Results represent the average of three independent experiments (mean \pm SD). Corresponding $P$ values analyzed by Spearman correlation test are indicated.

targets. Therefore, it was concluded that miR-409-3p inhibited growth, increased apoptosis and reduced invasion of LAD cells via inactivation of Akt signaling by targeting c-Met.

c-Met is upregulated and inversely correlated with miR-409-3p expression in human LAD tissues

To further examine the role of c-Met in LAD development, c-Met protein was detected in 34 cases of paired non-cancerous tissue (N) and LAD (T) tissue samples by Western blotting assay. The mean level of c-Met protein in LAD tissues was significantly higher than that in non-cancerous tissues ( $P=0.0054$; Fig. $10 \mathrm{~A}$ and B). Next, we investigated whether $c$-Met protein expression was inversely correlated with levels of miR-409-3p in LAD tissues. A total of 34 LAD tissues were analyzed for the expression levels of c-Met and for miR-409$3 p$ expression. A statistically significant inverse correlation was observed between c-Met and miR-409-3p ( $\mathrm{r}=-0.416 ; P=0.026$, Pearson's correlation; Fig. 10C). Therefore, these data further supported that downregulation of miR-409-3p was inversely correlated with overexpression of c-Met in LAD tissues.

\section{Discussion}

Emerging evidence has shown that dysregulation of miRNAs plays critical roles in tumorigenesis and the functions of miRNAs are complicated because they can downregulate numerous target genes including tumor suppressors and oncogenes [19]. Thus, it is necessary to explore their roles in tumor development. In the present study, we showed for the first time that miR-409-3p was significantly downregulated in LAD and an independent prognostic factor for patients. Functional analyses indicated that miR-409-3p 
could inhibit growth, induce apoptosis and reduce invasion in LAD cells via inactivation of Akt signaling by targeting c-Met. These data indicates that miR-409-3p may be a novel tumor suppressor which plays fundamental roles in lung carcinogenesis.

The pathogenesis of LAD involves multiple factors, including genetic predisposition, epigenetic regulation and environmental interaction [20]. The recent discovery of miRNAs and their epigenetic regulation add further complexity in human diseases and are attached more importance. Recently, correlations of aberrant expression of miRNAs and growth, metastasis and chemo- or radioresistance of LAD are increasingly reported. Kitamura' et al showed that miR-134/487b/655 cluster could regulate TGF- $\beta$-induced epithelial-mesenchymal transition and drug resistance to gefitinib by targeting MAGI2 in lung adenocarcinoma cells [21]. Zhao and his colleagues reported that downregulation of miR-145 might contribute to lung adenocarcinoma cell growth to form brain metastases [22]. Xiang' et al showed that microRNA-98 could sensitize cisplatin-resistant human lung adenocarcinoma cells by up-regulation of HMGA2 [23]. Liu and his colleagues reported that microRNA-449a could enhance radiosensitivity in CL1-0 lung adenocarcinoma cells [24]. However, the roles of miR-409-3p in LAD progression remain unclear and need to be further elucidated. First, we analyzed the correlation of miR-409-3p expression with clinicopathologic factors of LAD patients, and showed that the expression of miR-409-3p was closely correlated with tumor differentiation, pTNM stage and lymph node metastasis and poor survival of LAD patients. Further functional analysis indicated that transfection of miR-409-3p mimics could inhibit growth, induce apoptosis and inhibit migration or invasion in LAD cell. These data suggest that miR-409-3p functions as a tumor suppressor in LAD cells. Consistent with our work, other groups have reported that miR-409-3p functions as a tumor suppressor in gastric cancer. Zheng and his colleagues showed that microRNA-409-3p could suppress tumour cell invasion and metastasis by directly targeting radixin in gastric cancers [14]. Also, Li' et al reported that microRNA-409-3p could regulate cell proliferation and apoptosis by targeting PHF10 in gastric cancer [25]. In bladder cancer, microRNA409-3p is reported to inhibit migration and invasion of bladder cancer cells via targeting c-Met [13]. Moreover, miR-409-3p can inhibit HT1080 cell proliferation, vascularization and metastasis by targeting angiogenin [15]. In this report, we have, for the first time, established the role of miR-409-3p in LAD growth and invasion. Our findings, together with other results, indicated that miR-409-3p might function as a tumor suppressor in human cancers. Interestingly, Nadal and his groups identified a microRNA cluster at 14q32 drives aggressive lung adenocarcinoma, and further confirmed that eleven of 22 miRNAs associated with poor survival were encoded in a large miRNA cluster at 14q32, including 3 miRNAs encoded at 14q32 (miR-411, miR-370, and miR-376a) [26]. Although it is also originated from the chromosome 14q32 region, it acts as a tumor suppressor in LAD cells. However, little is known about the underlying molecular mechanisms of miR-409-3p in human LAD.

c-MET is a receptor tyrosine kinase that, after binding with its ligand, hepatocyte growth factor, activates a wide range of different cellular signaling pathways, including Akt signaling pathway [27]. To date, c-Met has been well-established as playing critical roles in growth, motility, migration and invasion $[28,29]$. c-Met has also been found to be aberrantly activated in human cancers via mutation, amplification or protein overexpression [30]. Previous study has reported that activation of hepatocyte growth factor-met autocrine loop enhances tumorigenicity in a human lung adenocarcinoma cell line [31]. Also, Stabile and his colleagues reported that transgenic mice overexpressing hepatocyte growth factor in the airways show increased susceptibility to lung cancer [32]. Interestingly, it is found that HGF/ c-MET system constitutes an autocrine activation loop in cancer-stromal myofibroblasts and this autocrine system may play a role in invasion and metastasis of lung adenocarcinoma [33]. Subsequent study shows that c-Met is directly downregulated by miR-449a, which inhibits growth and invasion of non-small cell lung cancer cells [34]. As one mRNA can be potentially regulated by multiple miRNAs [35], whether c-Met can be regulated by other miRNAs in human LAD needs to be further elucidated. In the present study, we confirmed that c-Met was a direct and functional target of miR-409-3p. A negative correlation between miR-409-3p 
and c-Met expression was observed in clinical LAD tissue samples. Transfection of miR-409$3 p$ mimics led to the decreased expression of c-Met protein in LAD cells, while transfection of miR-409-3p inhibitor induced the increased expression of c-Met protein. Luciferase activity assay indicated that miR-409-3p could bind to the 3'-UTR sequence of c-Met mRNA. Further functional studies indicated that siRNA-mediated c-Met downregulation could mimic the tumor suppressor roles of miR-409-3p and overexpression of c-Met could partially rescue the effects of miR-409-3p mimics on malignant phenotypes of LAD cells. Furthermore, we found that both upregulation of miR-409-3p and downregulation of c-Met could inactivate the Akt signaling and affect the expression of downstream effectors. Our data showed that miR-409-3p mimics and siRNA/c-Met could downregulate the expression of phosphorylated Akt protein, which eventually induced the decreased expression of Bcl-2, MMP-2 and MMP9 and the increased expression of Bax. The changes of those downstream effectors lead to growth inhibition, apoptosis enhancement and suppression of migration and invasion in LAD cells. Collectively, the downregulation of miR-409-3p in LAD may contribute to tumor growth and invasion, at least in part, via the upregulation of c-Met. Of course, this study has several limitations. First, while a single miRNA can target many genes, so other miRNA target validations should be performed in future researches. Second, further investigation of other LAD cell lines is needed to confirm the clinical significance of miR-409-3p in human LAD. Third, phenotypic analyses from pooled transfectants were performed, and phenotypic separation followed by differential expression analyses of miR409-3p and c-Met expression should be performed.

In conclusion, the present study shows for the first time that miR-409-3p is downregulated in LAD and may be an independent poor prognostic factor for patients. MiR409-3p possesses the potency to inhibit LAD growth, migration and invasion via regulation of Akt signaling pathway by targeting c-Met. The newly identified miR-409-3p/c-Met axis provides a novel insight into the pathogenesis of LAD, and provides us a wider perspective on LAD intervention and treatment.

\section{Acknowledgements}

This work was supported by grants from the National Natural Science Foundation of China (No.81272601,81472198), the Key Clinical Medicine Technology Foundation of Jiangsu Province (No.BL2014096), the Medical Key Talented Person Foundation of the Jiangsu Provincial Developing Health Project (No.RC2011080), Innovation Team Project of the Second Affiliated Hospital of Nanjing Medical University and "333 high class Talented Man Project" (No.2011-III-2630).

\section{References}

1 Jemal A, Siegel R, Ward E, Hao Y, Xu J, Thun MJ: Cancer statistics, 2009. CA Cancer J Clin 2009;59:225-249.

2 Gettinger S, Lynch T: A decade of advances in treatment for advanced non-small cell lung cancer. Clin Chest Med 2011;32:839-851.

3 Schrump DS, Nguyen DM: Targets for molecular intervention in multistep pulmonary carcinogenesis. World J Surg 2001;25:174-183.

4 Shivdasani RA: MicroRNAs: regulators of gene expression and cell differentiation. Blood 2006;108:36463653.

5 Pillai RS, Bhattacharyya SN, Filipowicz W: Repression of protein synthesis by miRNAs: how many mechanisms? Trends Cell Biol 2007;17:118-126.

6 Donadeu FX, Schauer SN, Sontakke SD: Involvement of miRNAs in ovarian follicular and luteal development. J Endocrinol 2012;215:323-334.

7 Liwak U, Faye MD, Holcik M: Translation control in apoptosis. Exp Oncol 2012;34: 218-230.

8 Rutnam ZJ, Yang BB: The involvement of microRNAs in malignant transformation. Histol Histopathol 2012;27:1263-1270. 
-9 Farazi TA, Hoell JI, Morozov P, Tuschl T: MicroRNAs in human cancer. Adv Exp Med Biol 2013;774:1-20.

10 Xie L, Yang Z, Li G, Shen L, Xiang X, Liu X, Xu D, Xu L, Chen Y, Tian Z, Chen X: Genome-wide identification of bone metastasis-related microRNAs in lung adenocarcinoma by high-throughput sequencing. PLoS One 2013;8:e61212.

11 Patnaik SK, Yendamuri S, Kannisto E, Kucharczuk JC, Singhal S, Vachani A: MicroRNA expression profiles of whole blood in lung adenocarcinoma. PLoS One 2012;7:e46045.

12 Zhang H, Su Y, Xu F, Kong J, Yu H, Qian B: Circulating MicroRNAs in Relation to EGFR Status and Survival of Lung Adenocarcinoma in Female Non-Smokers. PLoS One 2013;8:e81408.

13 Xu X, Chen H, Lin Y, Hu Z, Mao Y, Wu J, Xu X, Zhu Y, Li S, Zheng X, Xie L: MicroRNA-409-3p inhibits migration and invasion of bladder cancer cells via targeting c-Met. Mol Cell 2013;36:62-68.

14 Li C, Nie H, Wang M, Su L, Li J, Yu B, Wei M, Ju J, Yu Y, Yan M, Gu Q, Zhu Z, Liu B: MicroRNA-409-3p regulates cell proliferation and apoptosis by targeting PHF10 in gastric cancer. Cancer Lett 2012;320:189-197.

15 Weng C, Dong H, Chen G, Zhai Y, Bai R, Hu H, Lu L, Xu Z: miR-409-3p inhibits HT1080 cell proliferation, vascularization and metastasis by targeting angiogenin. Cancer Lett 2012;323:171-179.

16 Goetsch L, Caussanel V, Corvaia N: Biological significance and targeting of c-Met tyrosine kinase receptor in cancer. Front Biosci (Landmark Ed) 2013;18:454-473.

17 Cecchi F, Rabe DC, Bottaro DP: Targeting the HGF/Met signalling pathway in cancer. Eur J Cancer 2010;46:1260-1270.

18 Nam HJ, Chae S, Jang SH, Cho H, Lee JH: The PI3K-Akt mediates oncogenic Met-induced centrosome amplification and chromosome instability. Carcinogenesis 2010;31:1531-1540.

19 Chen PS, Su JL, Hung MC: Dysregulation of microRNAs in cancer. J Biomed Sci 2012;19:90.

20 Minna JD, Fong K, Zöchbauer-Müller S, Gazdar AF: Molecular pathogenesis of lung cancer and potential translational applications. Cancer J 2002;8:S41-46.

21 Kitamura K, Seike M, Okano T, Matsuda K, Miyanaga A, Mizutani H, Noro R, Minegishi Y, Kubota K, Gemma A: MiR-134/487b/655 Cluster Regulates TGF- $\beta$-induced Epithelial-Mesenchymal Transition and Drug Resistance to Gefitinib by Targeting MAGI2 in Lung Adenocarcinoma Cells. Mol Cancer Ther 2013 Dec 9. [Epub ahead of print]

22 Zhao C, Xu Y, Zhang Y, Tan W, Xue J, Yang Z, Zhang Y, Lu Y, Hu X: Downregulation of miR-145 contributes to lung adenocarcinoma cell growth to form brain metastases. Oncol Rep 2013;30:2027-2034.

-23 Xiang Q, Tang H, Yu J, Yin J, Yang X, Lei X: MicroRNA-98 sensitizes cisplatin-resistant human lung adenocarcinoma cells by up-regulation of HMGA2. Pharmazie 2013;68:274-281.

-24 Liu YJ, Lin YF, Chen YF, Luo EC, Sher YP, Tsai MH, Chuang EY, Lai LC: MicroRNA-449a enhances radiosensitivity in CL1-0 lung adenocarcinoma cells. PLoS One 2013;8:e62383.

25 Li C, Nie H, Wang M, Su L, Li J, Yu B, Wei M, Ju J, Yu Y, Yan M, Gu Q, Zhu Z, Liu B: MicroRNA-409-3p regulates cell proliferation and apoptosis by targeting PHF10 in gastric cancer. Cancer Lett 2012;320:189-197.

26 Nadal E, Zhong J, Lin J, Reddy RM, Ramnath N, Orringer MB, Chang AC, Beer DG, Chen G: A MicroRNA cluster at 14q32 drives aggressive lung adenocarcinoma. Clin Cancer Res 2014;20:3107-17.

27 Porter AC, Vaillancourt RR: Tyrosine kinase receptor-activated signal transduction pathways which lead to oncogenesis. Oncogene 1998;17:1343-1352.

28 Lowery FJ, Yu D: Growth factor signaling in metastasis: current understanding and future opportunities. Cancer Metastasis Rev 2012;31:479-491.

29 Grzelakowska-Sztabert B, Dudkowska M: Paradoxical action of growth factors: antiproliferative and proapoptotic signaling by HGF/c-MET. Growth Factors 2011;29:105-118.

-30 Shawna Leslie Organ: An overview of the c-MET signaling pathway. Ther Adv Med Oncol 2011;3:S7-S19.

-31 Yi S, Tsao MS: Activation of hepatocyte growth factor-met autocrine loop enhances tumorigenicity in a human lung adenocarcinoma cell line. Neoplasia 2000;2:226-234.

-32 Stabile LP, Lyker JS, Land SR, Dacic S, Zamboni BA, Siegfried JM: Transgenic mice overexpressing hepatocyte growth factor in the airways show increased susceptibility to lung cancer. Carcinogenesis 2006;27:15471555.

-33 Tokunou M, Niki T, Eguchi K, Iba S, Tsuda H, Yamada T, Matsuno Y, Kondo H, Saitoh Y, Imamura H, Hirohashi S: c-MET expression in myofibroblasts: role in autocrine activation and prognostic significance in lung adenocarcinoma. Am J Pathol 2001;158:1451-1463.

-34 Luo W, Huang B, Li Z, Li H, Sun L, Zhang Q, Qiu X, Wang E: MicroRNA-449a is downregulated in non-small cell lung cancer and inhibits migration and invasion by targeting c-Met. PLoS One 2013;8:e64759.

-35 Thomson DW, Bracken CP, Goodall GJ: Experimental strategies for microRNA target identification. Nucleic Acids Res 2011;39:6845-6853. 